\title{
New applications of the variational iteration method - from differential equations to $q$-fractional difference equations
}

\author{
Guo-Cheng $\mathrm{Wu}^{1,2^{*}}$ and Dumitru Baleanu $\mathrm{u}^{3,4,5^{*}}$
}

\author{
"Correspondence: \\ wuguocheng2002@yahoo.com.cn; \\ dumitru@cankaya.edu.tr \\ ${ }^{1}$ College of Mathematics \& \\ Information Science, Neijiang \\ Normal University, Neijiang, 641112, \\ China \\ ${ }^{3}$ Department of Mathematics and \\ Computer Sciences, Cankaya \\ University, Balgat, Ankara 06530, \\ Turkey \\ Full list of author information is \\ available at the end of the article
}

\begin{abstract}
The non-classical calculi such as $q$-calculus, fractional calculus and $q$-fractional calculus have been hot topics in both applied and pure sciences. Then some new linear and nonlinear models have appeared. This study mainly concentrates on the analytical aspects, and the variational iteration method is extended in a new way to solve an initial value problem.
\end{abstract}

MSC: $39 \mathrm{~A} 13 ; 74 \mathrm{H} 10$

Keywords: variational iteration method; fractional calculus; time scales; q-calculus; Laplace transform; symbolic computation

\section{Introduction}

Recently, $q$-fractional calculus has been paid much attention to [1-7], i.e., $q$-factional modeling, linear $q$-fractional systems, $q$-special functions etc. As is well known, both fractional calculus (FC) and $q$-calculus (QC) are not new as they appeared in 1695 and about 1920s, respectively. Fractional $q$-calculus (FQC) serves as a bridge between FC and QC. The early developments of $q$-fractional calculus can be found in [8-10]. Now, various $q$-fractional initial value problems are proposed in [3, 11-16].

The variational iteration method (VIM) [17-20] has been one of the often used nonlinear methods in initial boundary value problems of differential equations. In this study, the extension of the method into FQC is undertaken and the Caputo $q$-fractional initial value problems are investigated. Our study is organized as follows. In Section 2, the basic idea of the VIM is illustrated. In Section 3, the VIM is extended to $q$-difference equations, and the Lagrange multipliers of the method are presented for the equations of high-order $q$-derivatives. In Section 4, recent development of the method in fractional calculus is introduced. Following Section 4, the application of the VIM in $q$-fractional calculus is considered. Then the method is applied to the Caputo $q$-fractional initial value problem.

\section{The VIM in ordinary calculus}

We illustrate its basic idea through the following nonlinear system:

$$
\frac{d^{m} u}{d t^{m}}+R[u]+N[u]=g(t),
$$

( 2013 Wu and Baleanu; licensee Springer. This is an Open Access article distributed under the terms of the Creative Commons Attribution License (http://creativecommons.org/licenses/by/2.0), which permits unrestricted use, distribution, and reproduction in any medium, provided the original work is properly cited. 
where $u=u(t), R$ is a linear operator, $N$ is a nonlinear operator, $g(t)$ is a given continuous function and $d^{m} u / d t^{m}$ is the term of the highest-order derivative.

Then we construct the following correction function for Eq. (1):

$$
u_{n+1}=u_{n}+\int_{t_{0}}^{t} \lambda(t, \tau)\left(\frac{d^{m} u_{n}}{d \tau^{m}}+R\left[u_{n}\right]+N\left[u_{n}\right]-g(\tau)\right) d \tau
$$

where $\lambda=\lambda(t, \tau)$ is called the Lagrange multiplier which can be identified optimally by variational calculus and $u_{n}$ is the $n$th term approximate solution.

It is well known that Eq. (1) has the Lagrange multiplier [20]

$$
\lambda=\frac{(-1)^{m}(\tau-t)^{m-1}}{(m-1) !} .
$$

The interpretations and determination of various Lagrange multipliers can be found in the review article $[19,20]$.

Following the above steps, we can design a Maple-program which contains three parameters: ICs, Eqs and $n$. ICs reads the value of initial points. Eqs contains information of the linear terms, the nonlinear terms and the interval functions. $n$ means the approximate solution's truncated order.

Example 2.1 Consider the following Riccati equations [21]:

$$
\frac{d u}{d t}=1+2 u(t)-u^{2}(t), \quad u(0)=0 .
$$

We input ICs $:=[0,[0]], E q s:=\left[\left[u(t) \rightarrow-2 * u(t), u(t) \rightarrow u^{2}(t), t \rightarrow 1\right]\right], n$ can be set as $1,2, \ldots, 10$ and so on. We set $n=2$ and $n=9$, respectively. Since $u_{9}$ here is very tedious, we only give the analytical approximate solutions $u_{1}, u_{2}, u_{3}$ and $u_{4}$ as follows:

$$
\begin{aligned}
u_{1}= & t, \\
u_{2}= & t+t^{2}-\frac{1}{3} t^{3}, \\
u_{3}= & t+t^{2}+\frac{1}{3} t^{3}-\frac{1}{63} t^{7}+\frac{1}{9} t^{6}-\frac{1}{15} t^{5}-\frac{2}{3} t^{4}, \\
u_{4}= & t+t^{2}+1 / 3 t^{3}+\frac{71}{315} t^{7}+\frac{4}{45} t^{6}-3 / 5 t^{5}-1 / 3 t^{4}-\frac{1}{59,535} t^{15}+\frac{1}{3,969} t^{14} \\
& -\frac{41}{36,855} t^{13}-\frac{1}{1,890} t^{12}+\frac{27}{1,925} t^{11}-\frac{62}{4,725} t^{10}-\frac{62}{945} t^{9}+\frac{17}{420} t^{8} .
\end{aligned}
$$

The comparisons between $u_{2}, u_{9}$ and the exact solution are listed in Table 1 .

Example 2.2 The second example is a system representing a nonlinear reaction [22]:

$$
\left\{\begin{array}{lrl}
\frac{d u}{d t}=-u, & u(0)=1, \\
\frac{d v}{d t}=u-v^{2}, & v(0)=0, \\
\frac{d w}{d t}=v^{2}, & w(0)=0 .
\end{array}\right.
$$


Table 1 Comparisons between $u_{2}, u_{9}$ and the exact solution

\begin{tabular}{|c|c|c|c|}
\hline$t$ & $\operatorname{VIM}\left[u_{2}\right]$ & $\operatorname{VIM}\left[u_{9}\right]$ & Exact solution \\
\hline 0 & 0 & 0 & 0 \\
\hline 0.1 & 0.109666666667 & 0.110295196915 & 0.110295196918 \\
\hline 0.2 & 0.237333333333 & 0.241976799617 & 0.241976799622 \\
\hline 0.3 & 0.381000000000 & 0.395104848537 & 0.395104848660 \\
\hline 0.4 & 0.538666666667 & 0.567812165419 & 0.567812166292 \\
\hline 0.5 & 0.708333333333 & 0.756014390810 & 0.756014393428 \\
\hline 0.6 & 0.888000000000 & 0.953566212412 & 0.953566216469 \\
\hline 0.7 & 1.075666666667 & 1.15294896351 & 1.15294896698 \\
\hline 0.8 & 1.26933333333 & 1.34636365376 & 1.34636365537 \\
\hline 0.9 & 1.46700000000 & 1.52691131294 & 1.52691131327 \\
\hline 1.0 & 1.66666666667 & 1.68949839169 & 1.68949839159 \\
\hline
\end{tabular}

The information of the system (5) reads

$$
\begin{aligned}
I C s:= & {[0,[1],[0],[0]] } \\
\text { Eqs }:= & {\left[[(u, v, w) \rightarrow u,(u, v, w) \rightarrow 0, t \rightarrow 0],\left[(u, v, w) \rightarrow-u+v^{2},(u, v, w) \rightarrow 0, t \rightarrow 0\right],\right.} \\
& {\left.\left[(u, v, w) \rightarrow v^{2},(u, v, w) \rightarrow 0, t \rightarrow 0\right]\right] ; }
\end{aligned}
$$

$n:=4$.

The fourth-order approximation can be presented as

$$
\begin{aligned}
u_{4}:= & 1-t+1 / 2 t^{2}-1 / 6 t^{3}+1 / 24 t^{4}, \\
v_{4}:= & t-\frac{1}{2} t^{2}-\frac{1}{6} t^{3}+\frac{1}{126} t^{7}-1 / 9 t^{6}+\frac{1}{60} t^{5}+\frac{5}{24} t^{4} \\
& -\frac{1}{59,535} t^{15}-\frac{1}{7,938} t^{14}-\frac{1}{29,484} t^{13} \\
& +\frac{13}{9,072} t^{12}+\frac{47}{33,264} t^{11}-\frac{23}{3,024} t^{10}-\frac{59}{9,072} t^{9}+\frac{5}{144} t^{8}, \\
w_{4}:= & \frac{1}{3} t^{3}-\frac{1}{126} t^{7}+1 / 9 t^{6}-\frac{1}{60} t^{5}-\frac{1}{4} t^{4}+\frac{1}{59,535} t^{15} \\
& +\frac{1}{7,938} t^{14}+\frac{1}{29,484} t^{13}-\frac{13}{9,072} t^{12} \\
& -\frac{47}{33,264} t^{11}+\frac{23}{3,024} t^{10}+\frac{59}{9,072} t^{9}-\frac{5}{144} t^{8} .
\end{aligned}
$$

We can calculate $\left[u_{100}, v_{100}, w_{100}\right],\left[u_{200}, v_{200}, w_{200}\right],\left[u_{300}, v_{300}, w_{300}\right]$ even higher-order approximation. Noting that $u_{n}$ tends to $e^{-t}$ for $n \rightarrow \infty$, we only compare $\left[v_{4}, w_{4}\right]$ and $\left[v_{8}, w_{8}\right]$ with the numerical results from the Runge-Kutta method (RKM) in Figure 1 and Figure 2, respectively. Obviously, $v_{8}$ and $w_{8}$ have higher accuracies than $v_{4}$ and $w_{4}$. With symbolic computation, if the computer is excellent enough, higher accuracies can be obtained.

\section{The VIM in q-calculus}

The $q$-derivative is a deformation of the classical derivative and it has played a crucial role in statistical physics and quantum mechanics. Let us revisit some properties of $q$-calculus [23-25]. 


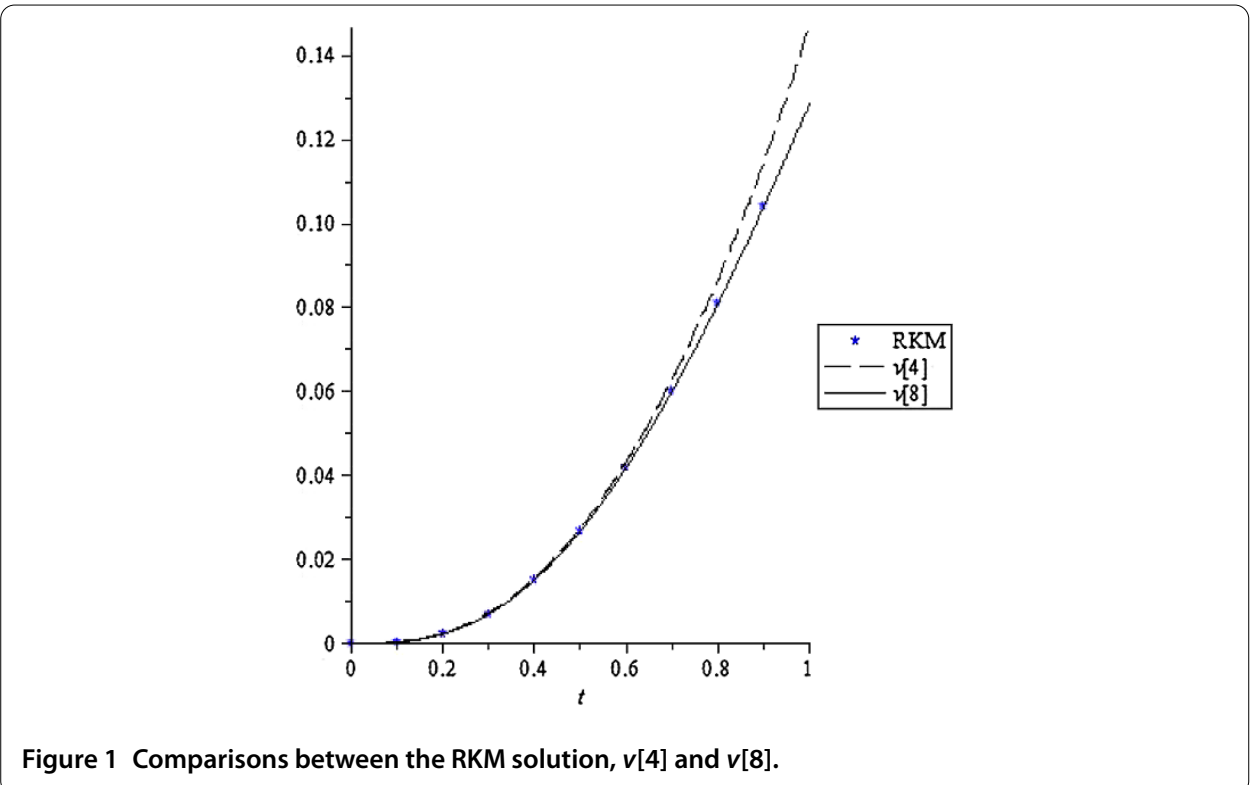

Figure 1 Comparisons between the RKM solution, $v[4]$ and $v[8]$.

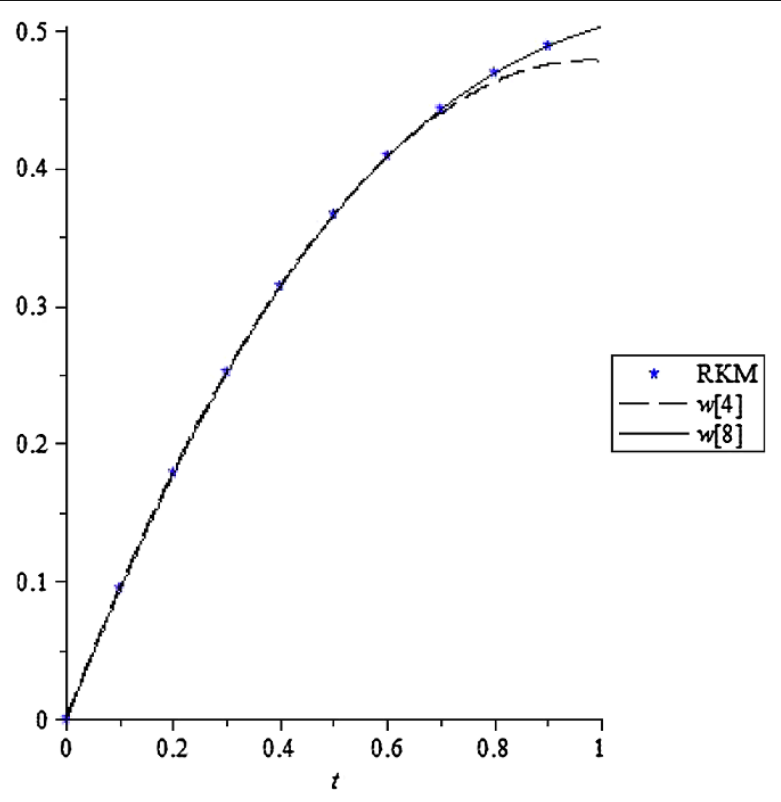

Figure 2 Comparisons between the RKM solution, $w[4]$ and $w[8]$.

Definition 3.1 ( $q$-calculus) Let $f(x)$ be a real continuous function. The $q$-derivative is defined by

$$
\frac{d_{q}}{d_{q} x} f(x)=\frac{f(q x)-f(x)}{(q-1) x}, \quad x \neq 0,0<q<1
$$

and

$$
\left.\frac{d_{q}}{d_{q} x} f(x)\right|_{x=0}=\lim _{n \rightarrow \infty} \frac{f\left(q^{n}\right)-f(0)}{q^{n}} .
$$


The partial $q$-derivative is defined as

$$
\frac{\partial_{q}}{\partial_{q} x} f(x ; y ; \ldots)=\frac{f(q x ; y ; \ldots)-f(x ; y ; \ldots)}{(q-1) x} .
$$

Jackson's $q$-integral $[26,27]$ is given as

$$
\int_{0}^{x} f(t) d_{q} t=(1-q) x \sum_{0}^{\infty} q^{n} f\left(q^{n} x\right) .
$$

Property 3.2 q-Leibniz product law is

$$
\frac{d_{q}}{d_{q} x}[g(x) f(x)]=g(q x) \frac{d_{q}}{d_{q} x} f(x)+f(x) \frac{d_{q}}{d_{q} x} g(x) .
$$

Property 3.3 $q$-integration by parts holds

$$
\int_{a}^{b} g(q t) \frac{d_{q}}{d_{q} t} f(t) d_{q} t=\left.[f(t) g(t)]\right|_{a} ^{b}-\int_{a}^{b} f(t) \frac{d_{q}}{d_{q} t} g(t) d_{q} t .
$$

The properties above are needed in the construction of the correction functional for $q$-difference equations. For more results and properties in $q$-calculus, readers are referred to monographs [23-25].

Lemma 3.4 ([28]) For the first-order q-difference equation,

$$
\frac{d_{q} x}{d_{q} t}+f(t, x)=0
$$

one of the Lagrange multipliers is $\lambda(t, \tau)=-1$.

Lemma 3.5 ([29]) For the q-difference equation of second order,

$$
\frac{d_{q}^{2} x}{d_{q} t^{2}}+f(t, x)=0
$$

one of the Lagrange multipliers can be identified as

$$
\lambda(t, \tau)=q^{-1}(\tau-t q) .
$$

Proof We revisit the proof in [29]. First, establish the correctional functional for Eq. (11) as

$$
x_{n+1}=x_{n}+\int_{0}^{t} \lambda\left(t, q^{2} \tau\right)\left(\frac{d_{q}^{2} x_{n}}{d_{q} \tau^{2}}+f\left(\tau, x_{n}\right)\right) d_{q} \tau .
$$

We only use the leading term $\frac{d_{q}^{2} x}{d_{q} t^{2}}$, while other terms are restricted variations

$$
x_{n+1}=x_{n}+\int_{0}^{t} \lambda\left(t, q^{2} \tau\right)\left(\frac{d_{q}^{2} x_{n}}{d_{q} \tau^{2}}+f\left(\tau, x_{n}\right)\right) d_{q} \tau .
$$


Through the integration by parts (9), we can have

$$
\delta x_{n+1}=\left(1-\left.q \frac{\partial_{q}}{\partial_{q} \tau} \lambda(t, \tau)\right|_{\tau=t}\right) \delta x_{n}+\left.\lambda(t, q \tau)\right|_{\tau=t} \delta x_{n}^{\prime}-q \int_{0}^{t} \frac{\partial_{q}^{2} \lambda(t, \tau)}{\partial_{q} \tau^{2}} \delta x_{n} d_{q} \tau,
$$

where $\delta$ is the variation operator and " denotes the $q$-derivative with respect to $t$. As a result, the system of the Lagrange multiplier can be obtained:

the coefficient of $\delta x_{n}: 1-\left.q \frac{\partial_{q} \lambda(t, \tau)}{\partial_{q} \tau}\right|_{\tau=t}=0$,

the coefficient of $\delta x_{n}^{\prime}(x, \tau):\left.\lambda(t, q \tau)\right|_{\tau=t}=0$,

and the coefficient of $\delta x_{n}$ in the $q$-integral: $q \frac{\partial_{q}^{2} \lambda(t, \tau)}{\partial_{q} \tau^{2}}=0$,

from which we can get

$$
\lambda(t, \tau)=q^{-1}(\tau-t q) .
$$

Furthermore, Kong [30] gave the Lagrange multiplier for the $q$-difference equations of third order,

$$
\lambda(t, \tau)=-q^{-3} \frac{(\tau-t q)\left(\tau-t q^{2}\right)}{[2]_{q} !},
$$

where $[k]_{q}$ ! denotes the $q$-factorial and $[k]_{q} !=1(1+q)\left(1+q+q^{2}\right) \cdots\left(1+q+\cdots+q^{k-1}\right)$ for the integer $k$.

More generally, one can derive the following Theorem 3.6.

Theorem 3.6 For the q-difference equation of mth order,

$$
\frac{d_{q}^{m} x}{d_{q} t^{m}}+f(t, x)=0
$$

establish the correctional functional for Eq. (17) as

$$
x_{n+1}=x_{n}+\int_{0}^{t} \lambda\left(t, q^{m} \tau\right)\left(\frac{d_{q}^{m} x_{n}}{d_{q} \tau^{m}}+f\left(\tau, x_{n}\right)\right) d_{q} \tau
$$

If $f\left(\tau, x_{n}\right)$ is considered as a restricted variation, one can derive a $q$-analogue Lagrange multiplier

$$
\lambda(t, \tau)=q^{-\frac{m(m-1)}{2}} \frac{(-1)^{m}(\tau-t q)^{(m-1)}}{[m-1]_{q} !}
$$

or

$$
\lambda\left(t, q^{m} \tau\right)=-\frac{(t-\tau q)^{(m-1)}}{[m-1]_{q} !},
$$

where $(t-a)^{(m-1)}=(t-a)(t-q a) \cdots\left(t-q^{m-1} a\right)$ for the integer $m$. 
Substituting (20) into (18), one can obtain a $q$-variational iteration formula

$$
x_{n+1}=x_{n}-\int_{0}^{t} \frac{(t-\tau q)^{(m-1)}}{[m-1]_{q} !}\left(\frac{d_{q}^{m}}{d_{q} \tau^{m}} x_{n}+f\left(\tau, x_{n}\right)\right) d_{q} \tau \text {. }
$$

Here the initial iteration value can be determined via the $q$-Taylor series [23].

Example 3.7 Consider the simple linear $q$-difference equation of second order [29]

$$
\frac{d_{q}^{2} u(t)}{d_{q} t^{2}}-u(t)=0
$$

subject to the initial conditions

$$
u(0)=1 \quad \text { and } \quad \frac{d_{q} u(0)}{d_{q} t}=1 .
$$

The iteration formula and the initial iteration can be determined as

$$
\left\{\begin{array}{l}
u_{n+1}=u_{n}+\frac{1}{q} \int_{0}^{t}\left(q^{2} \tau-q t\right)\left[\frac{d_{q}^{2} u_{n}(\tau)}{d_{q} \tau^{2}}-u_{n}(\tau)\right] d_{q} \tau \\
u_{0}=1+\frac{t}{[1]_{q} !}
\end{array}\right.
$$

The successive solution can be given as

$$
\begin{aligned}
u_{0}= & 1+\frac{t}{[1]_{q} !}, \\
u_{1}= & 1+\frac{t}{[1]_{q} !}+\frac{t^{2}}{[2]_{q} !}+\frac{t^{3}}{[3]_{q} !}, \\
& \vdots \\
u_{n}= & \sum_{k=0}^{2 n+1} \frac{t^{k}}{[k]_{q} !} .
\end{aligned}
$$

Recall that the limit $u=\lim _{t \rightarrow \infty} u_{n}=e_{q}(t)$ is an exact solution of (22). Here $e_{q}(t)$ is one of the $q$-exponential functions.

\section{The VIM in fractional calculus}

Let $u(t)$ be a real-valued function defined on a closed interval $[a, b]$.

Definition 4.1 The R-L integration of $\alpha$ order is defined as

$$
{ }_{0} I_{t}^{\alpha} u(t)=\frac{1}{\Gamma(\alpha)} \int_{a}^{t}(t-\tau)^{\alpha-1} u(\tau) d \tau, \quad 0<t, 0<\alpha .
$$

Definition 4.2 The left Caputo derivative is defined by

$$
{ }_{0}^{C} D_{t}^{\alpha} u(t)=\frac{1}{\Gamma(m-\alpha)} \int_{0}^{t} \frac{1}{(t-\tau)^{\alpha-m+1}} \frac{d^{m} u(\tau)}{d \tau^{m}} d \tau, \quad 0<t, 0<\alpha, m=[\alpha]+1 .
$$


Definition 4.3 The $\alpha$ th Riemann-Liouville (R-L) derivative of a function $u(t)$ is defined by

$$
{ }_{0}^{R L} D_{t}^{\alpha} u=\frac{1}{\Gamma(m-\alpha)} \frac{d^{m}}{d t^{m}} \int_{0}^{t} \frac{1}{(t-\tau)^{\alpha-m+1}} u(\tau) d \tau, \quad 0<t, 0<\alpha, m=[\alpha]+1 .
$$

More results and properties can be found in [31,32].

In Sections 2 and 3, we note that the integration by parts plays an important role and is often used in the derivation of the Lagrange multipliers in ordinary calculus. But in FC, the similar integration by parts cannot hold. That's the main reason why the applications of the VIM were not very successful for fractional differential equations (FDEs). The popular iteration formulae of the VIM directly employed the so-called Lagrange multiplier $\lambda(t, \tau)=-1$. For the generalized FDE

$$
{ }_{0}^{C} D_{t}^{\alpha} u+R[u]+N[u]=f(t)
$$

the variational iteration formula was suggested

$$
u_{n+1}=u_{n}+\int_{0}^{t}(-1)\left({ }_{0}^{C} D_{\tau}^{\alpha} u_{n}+R\left[u_{n}\right]+N\left[u_{n}\right]-f(\tau)\right) d \tau .
$$

One can check the formula (26) results in a poor convergence even for a linear FDE. Such difficulty can be overcome by the Laplace transform [31-33]. The following iteration formula is initially proposed in $[34,35]$. Let us revisit the proof.

Theorem 4.4 For the generalized FDE, one can have the variational iteration formula

$$
u_{n+1}=u_{n}+\int_{0}^{t} \lambda(t, \tau)\left({ }_{0}^{C} D_{\tau}^{\alpha} u_{n}+R\left[u_{n}\right]+N\left[u_{n}\right]-f(\tau)\right) d \tau, \quad 0<\alpha,
$$

where the function $\lambda(t, \tau)=(-1)^{\alpha}(\tau-t)^{\alpha-1} / \Gamma(\alpha)$ is a Lagrange multiplier for any order $\alpha$.

Proof We can construct a correction functional through the R-L integration

$$
u_{n+1}=u_{n}+{ }_{0} I_{t}^{\alpha} \lambda(t, \tau)\left[{ }_{0}^{C} D_{\tau}^{\alpha} u_{n}+R\left[u_{n}\right]+N\left[u_{n}\right]-f(\tau)\right] .
$$

Take the Laplace transform $L$ to both sides of (28)

$$
\bar{u}_{n+1}(s)=\bar{u}_{n}(s)+L\left[{ }_{0} I_{t}^{\alpha} \lambda(t, \tau)\left({ }_{0}^{C} D_{\tau}^{\alpha} u_{n}+R\left[u_{n}\right]+N\left[u_{n}\right]-f(\tau)\right)\right],
$$

where $\bar{u}_{n}(s)=L\left[u_{n}(t)\right]$.

Assuming the terms $R\left[u_{n}\right]$ and $N\left[u_{n}\right]$ are restricted variations, respectively, we only need to consider the term

$$
{ }_{0} I_{t}^{\alpha} \lambda_{0}^{C} D_{t}^{\alpha} u_{n}=\frac{1}{\Gamma(\alpha)} \int_{0}^{t}(t-\tau)^{\alpha-1} \lambda(t, \tau){ }_{0}^{C} D_{\tau}^{\alpha} u_{n}(\tau) d \tau .
$$


Setting the Lagrange multiplier $\lambda(t, \tau)=\left.\lambda(X)\right|_{X=t-\tau}$, Eq. (30) can be considered as a convolution of the function $a(t)=\lambda(t) t^{\alpha-1} / \Gamma(\alpha)$ and the term ${ }_{0}^{C} D_{t}^{\alpha} u_{n}(t)$.

Making the correction functional of Eq. (29) stationary, we can get

$$
\delta \bar{u}_{n+1}(s)=\delta \bar{u}_{n}(s)+\delta\left[\bar{a}(s) s^{\alpha} \bar{u}_{n}(s)-\sum_{k=0}^{m-1} u^{(k)}\left(0^{+}\right) s^{\alpha-1-k}\right]=\left(1+\bar{a}(s) s^{\alpha}\right) \delta \bar{u}_{n}(s) .
$$

From Eq. (16), with the inverse Laplace transform $L^{-1}$, we can have

$$
a(t)=L^{-1}[\bar{a}(s)]=-\frac{t^{\alpha-1}}{\Gamma(\alpha)}, \quad 0<\alpha .
$$

For $a(t)=\lambda(t) t^{\alpha-1} / \Gamma(\alpha)$, the Lagrange multiplier can be explicitly identified as

$$
\lambda(t, \tau)=-1 .
$$

As a result, the iteration formula is given as

$$
u_{n+1}=u_{n}-{ }_{0} I_{t}^{\alpha}\left[{ }_{0}^{C} D_{\tau} u_{n}+R\left[u_{n}\right]+N\left[u_{n}\right]-f(\tau)\right], \quad 0<\alpha .
$$

This completes the proof.

We only derive the simplest Lagrange multiplier $\lambda(t, \tau)=-1$ here. In fact, more explicit Lagrange multipliers can be identified if more terms in $R\left[u_{n}\right]$ (if they exist) are used. For example, we can derive a variational iteration formula

$$
\begin{aligned}
u_{n+1}= & u_{n}-\int_{0}^{t}(t-\tau)^{\gamma-1} E_{\gamma-\beta, \gamma}\left(-(t-\tau)^{\gamma-\beta}\right) \\
& \times\left[{ }_{0}^{C} D_{\tau}^{\gamma} u_{n}+{ }_{0}^{C} D_{\tau}^{\beta} u_{n}+f\left(\tau, u_{n}\right)\right] d \tau, \quad 0<\beta<\gamma
\end{aligned}
$$

for a multi-order FDE

$$
{ }_{0}^{C} D_{t}^{\gamma} u+{ }_{0}^{C} D_{t}^{\beta} u+f(t, u)=0, \quad 0<t, 0<\beta<\gamma .
$$

For $\gamma=1, \beta=0$ and $\gamma=2, \beta=0$, Eq. (35) reduces to the formulae (see the iteration formulae (19a) and (21a) in [20])

$$
\left\{\begin{array}{l}
u_{n+1}=u_{n}+\int_{0}^{t} \lambda(t, \tau)\left(u_{n}^{\prime}+u_{n}+f\left(\tau, u_{n}\right)\right) d \tau, \\
\lambda(t, \tau)=-\left.(t-\tau)^{\gamma-1} E_{\gamma-\beta, \gamma}\left(-(t-\tau)^{\gamma-\beta}\right)\right|_{\gamma=1, \beta=0}=-e^{-(t-\tau)}
\end{array}\right.
$$

and

$$
\left\{\begin{array}{l}
u_{n+1}=u_{n}+\int_{0}^{t} \lambda(t, \tau)\left(u_{n}^{(2)}+u_{n}+f\left(\tau, u_{n}\right)\right) d \tau \\
\lambda=-\left.(t-\tau)^{\gamma-1} E_{\gamma-\beta, \gamma}\left(-(t-\tau)^{\gamma-\beta}\right)\right|_{\gamma=2, \beta=0}=\sin (\tau-t) .
\end{array}\right.
$$


We conclude the following useful Lagrange multipliers for FDEs
I. $\left\{\begin{array}{l}{ }_{0}^{C} D_{t}^{\gamma} u+f\left(t,{ }_{0}^{C} D_{t}^{\beta} u, u\right)=0, \quad 0<\beta<\gamma, \\ \lambda(t, \tau)=\frac{\left.(-1)^{\gamma}(\tau-t)\right)^{\gamma-1}}{(\gamma-1) !},\end{array}\right.$
II. $\left\{\begin{array}{l}{ }_{0}^{C} D_{t}^{\gamma} u+{ }_{0}^{C} D_{t}^{\beta} u+f(t, u)=0, \quad 0<\beta<\gamma, \\ \lambda(t, \tau)=-(t-\tau)^{\gamma-1} E_{\gamma-\beta, \gamma}\left(-(t-\tau)^{\gamma-\beta}\right),\end{array}\right.$
III. $\left\{\begin{array}{l}{ }_{0}^{R L} D_{t}^{\gamma} u+f\left(t,{ }_{0}^{C} D_{t}^{\beta} u, u\right)=0, \quad 0<\beta<\gamma, \\ \lambda(t, \tau)=\frac{(-1)^{\gamma}(\tau-t)^{\gamma-1}}{(\gamma-1) !}\end{array}\right.$

and

IV. $\left\{\begin{array}{l}{ }_{0}^{R L} D_{t}^{\gamma} u+{ }_{0}^{C} D_{t}^{\beta} u+f(t, u)=0, \quad 0<\beta<\gamma, \\ \lambda(t, \tau)=-(t-\tau)^{\gamma-1} E_{\gamma-\beta, \gamma}\left(-(t-\tau)^{\gamma-\beta}\right) .\end{array}\right.$

Example 4.5 Let us consider the linear fractional Schrodinger equation [36]

$$
i_{0}^{C} D_{t}^{\alpha} u+\frac{1}{2} \frac{\partial^{2} u}{\partial x^{2}}=0, \quad u(x, 0)=e^{i x} .
$$

The variational iteration formula for (40) reads

$$
u_{n+1}=u_{n}-{ }_{0} I_{t}^{\alpha}\left(i_{0}^{C} D_{\tau}^{\alpha} u_{n}+\frac{1}{2} \frac{\partial^{2} u_{n}}{\partial x^{2}}\right) .
$$

Starting from the initial iteration $u_{0}=u(x, 0)=e^{i x}$, the successive approximate solutions can be given as

$$
\begin{aligned}
u_{1}= & e^{i x}+e^{i x} \frac{i t^{\alpha}}{2 \Gamma(1+\alpha)}, \\
u_{2}= & e^{i x}+e^{i x} \frac{i t^{\alpha}}{2 \Gamma(1+\alpha)}-e^{i x} \frac{t^{\alpha}}{4 \Gamma(1+\alpha)}, \\
& \vdots \\
u_{n}= & e^{i x} \sum_{k=0}^{n} \frac{(i t)^{k \alpha}}{2^{k} \Gamma(1+k \alpha)} .
\end{aligned}
$$

For $n \rightarrow \infty, u_{n}$ tends to $e^{i x} E_{\alpha}\left(i t^{\alpha} / 2\right)$ which is an exact solution of (41).

Example 4.6 As the second example, consider the nonlinear fractional Schrodinger equation [36]

$$
i_{0}^{C} D_{t}^{\alpha} u+\frac{1}{2} \frac{\partial^{2} u}{\partial x^{2}}+|u|^{2} u=0, \quad u(x, 0)=e^{i x} .
$$

The corresponding iteration formula reads

$$
u_{n+1}=u_{n}-{ }_{0} I_{t}^{\alpha}\left(i_{0}^{C} D_{\tau}^{\alpha} u_{n}+\frac{1}{2} \frac{\partial^{2} u_{n}}{\partial x^{2}}+\left|u_{n}\right|^{2} u_{n}\right) .
$$


As a result, the approximate solutions can be obtained

$$
\begin{aligned}
u_{0}= & e^{i x} \\
u_{1}= & e^{i x}\left(1-\frac{1}{2} \frac{t^{\alpha}}{\Gamma(1+\alpha)}\right), \\
u_{2}= & e^{i x}\left(1-\frac{1}{2} \frac{t^{\alpha}}{\Gamma(1+\alpha)}+\frac{5}{4} \frac{t^{2 \alpha}}{\Gamma(1+2 \alpha)}-\frac{3}{4} \frac{\Gamma(1+2 \alpha) t^{3 \alpha}}{\Gamma^{2}(1+\alpha) \Gamma(1+3 \alpha)}\right. \\
& \left.+\frac{1}{8} \frac{\Gamma(1+3 \alpha) t^{4 \alpha}}{\Gamma^{3}(1+\alpha) \Gamma(1+4 \alpha)}\right), \\
& \vdots
\end{aligned}
$$

Remarks Our simplest iteration formula (34) can reduce to the Volterra integral equation. See the analysis of the convergence and existence in [37] and the references therein. However, regarding Eq. (36), the VIM transforms it into a more general Volterra integral equation from which one can obtain approximate solutions of higher accuracies.

FDEs have been proven to be a useful tool to describe the nonlocal behaviors or long range interactions of dynamical systems. The previous applications of the VIM just 'guessed' the Lagrange multipliers or directly used the one in ordinary differential equations. In this study, various Lagrange multipliers are identified more explicitly and the variational approach for FDEs is systematically developed now.

\section{The Caputo $q$-fractional initial value problem}

We employ some notations of the $q$-fractional derivative and integral in [38]. For $0<q<1$, let $T_{q}$ be the time scale

$$
T_{q}=\left\{q^{m}: m \in \mathrm{Z}\right\} \cup\{0\}
$$

where $\mathrm{Z}$ is the set of integers.

Definition 5.1 More generally, if $\alpha$ is a nonnegative real number, then we define the time scale as follows:

$$
T_{q}^{\alpha}=\left\{q^{m+\alpha}: m \in Z\right\} \cup\{0\}
$$

The $q$-fractional derivative and integral have been defined in earlier work [8-10].

Definition 5.2 The $q$-fractional integral of $\alpha$ order is defined by

$$
{ }_{q} I_{0}^{\alpha} f(t)=\frac{1}{\Gamma_{q}(\alpha)} \int_{0}^{t}(t-q \tau)^{(\alpha-1)} f(\tau) d_{q} \tau, \quad t \in T_{q}^{\alpha}
$$

and the left Caputo $q$-fractional derivative is defined as

$$
{ }_{q}^{C} D_{0}^{\alpha} u(t)=\frac{1}{\Gamma(m-\alpha)} \int_{0}^{t} \frac{1}{(t-\tau)^{(\alpha-m+1)}} \frac{d^{m}}{d \tau^{m}} u(\tau) d \tau, \quad 0<\alpha, m=[\alpha]+1, t \in T_{q}^{\alpha} .
$$


When $v$ is not a positive integer, the $q$-factorial function is defined by

$$
(t-\tau)^{(v)}=t^{v} \prod_{n=0}^{\infty} \frac{1-\frac{q^{n} \tau}{t}}{1-\frac{q^{\nu+n} \tau}{t}}
$$

The fractional $q$-derivative of the $q$-factorial function with respect to $t$ is

$$
{ }_{q}^{C} D_{a}^{\alpha}(t-a)^{(v)}=\frac{\Gamma_{q}(v+1)}{\Gamma_{q}(v-\alpha+1)}(t-a)^{(v-\alpha)}
$$

and

$$
{ }_{q} I_{a}^{\alpha}(t-a)^{(v)}=\frac{\Gamma_{q}(v+1)}{\Gamma_{q}(\alpha+v+1)}(t-a)^{(\alpha+v)}, \quad a<t
$$

where $\alpha \in R^{+}$and $v \in(-1, \infty)$.

Now, we introduce the $q$-Laplace transform and some properties.

Definition 5.3 The $q$-Laplace transform was defined by Hahn [39] in 1949 as follows:

$$
L_{q}[h(t)]=\frac{1}{1-q} \int_{0}^{1 / s} h(t) e_{2, q}(q s t) d_{q} t
$$

where $e_{2, q}(t)=\prod_{n=0}^{\infty}\left(1-q^{n} t\right), e_{2, q}(0)=1$.

Lemma 5.4 ([38]) Let $g(t)$ be an analytic function and assume $g(t)=t^{\nu-1}$ on $T_{q} \backslash\{0\}$, where $v \in R \backslash\{\ldots,-2,-1,0\}$. Then the following convolution theorem can hold:

$$
L_{q}[h(t) * g(t)]=L_{q}[h(t)] L_{q}[g(t)]
$$

where the convolution is defined as

$$
(h * g)(s)=\frac{1}{1-q} \int_{0}^{s} h(\tau) g[s-q \tau] d_{q} \tau \quad \text { and } \quad g[s-q \tau]=(s-q \tau)^{(\nu-1)} .
$$

Lemma 5.5 For the Caputo q-derivative of $g(t)$, the following Laplace transform holds:

$$
L_{q}\left[{ }_{q}^{C} D_{0}^{\alpha} g(t)\right]=\frac{s^{\alpha}}{(1-q)^{\alpha}} L_{q}[g(t)]-\sum_{i=0}^{m-1} \frac{d_{q}^{i} g(0)}{d_{q} t^{i}} \frac{s^{\alpha-i-1}}{(1-q)^{\alpha-i}}, \quad 0<\alpha, m=[\alpha]+1
$$

and

$$
L_{q}\left[{ }_{q} I_{0}^{\alpha} g(t)\right](s)=\frac{(1-q)^{\alpha}}{s^{\alpha}} L_{q}[g(t)](s) .
$$

Lemma $5.6([38])(t-\tau)^{(\beta+\gamma)}=(t-\tau)^{(\beta)}\left(t-q^{\beta} \tau\right)^{(\gamma)}, \beta, \gamma \in R$.

The existence and uniqueness of the solutions of the Caputo $q$-initial value problems have been discussed in [40]. 
Lemma 5.7 Considering the initial value problems of the Caputo q-fractional equations,

$$
{ }_{q}^{C} D_{0}^{\alpha} u(t)+f(t, u)=0, \quad \frac{d_{q}}{d_{q} t} u_{i}(0)=a_{i}, \quad m=[\alpha]+1, i=0, \ldots, m-1,
$$

we construct a q-fractional correction functional

$$
u_{n+1}=u_{n}+{ }_{q} I_{0}^{\alpha} \lambda(t, \tau)\left[{ }_{q}^{C} D_{0}^{\alpha} u_{n}+f\left(\tau, u_{n}\right)\right]
$$

One of the Lagrange multipliers can be identified as $\lambda(t, \tau)=-1$.

Proof Take the Laplace transform of both sides of (54)

$$
\begin{aligned}
L_{q}\left[u_{n+1}\right]= & L_{q}\left[u_{n}\right]+(1-q) L_{q}\left[\frac{1}{(1-q)} \int_{0}^{t} \frac{(t-q \tau)^{(\alpha-1)}}{\Gamma_{q}(\alpha)} \lambda(t, \tau)\right. \\
& \left.\times\left[{ }_{q}^{C} D_{0}^{\alpha} u_{n}+f\left(\tau, u_{n}\right)\right]\right] d_{q} \tau .
\end{aligned}
$$

From Lemma 5.6, we set $\lambda(t, \tau)=\sum_{i=0}^{\infty} a_{i}\left[t-q^{\alpha} \tau\right]^{\left(\beta_{i}\right)}$, where $\beta_{i} \geq 0$. Then the Lagrange multiplier is 'good' enough so that the product of $\frac{(t-q \tau)^{(\alpha-1)}}{\Gamma_{q}(\alpha)}$ and $\lambda(t, \tau)$ is similar as the function $g[s-q \tau]$ in (51b) and $\left.\frac{1}{(1-q)} \int_{0}^{t} \frac{(t-q \tau)^{(\alpha-1)}}{\Gamma_{q}(\alpha)} \lambda(t, \tau){ }_{q}^{C} D_{0}^{\alpha} u_{n}+f\left(\tau, u_{n}\right)\right]$ becomes a convolution (51b).

Then we get the following equation:

$$
\begin{aligned}
L_{q}\left[u_{n+1}\right]= & L_{q}\left[u_{n}\right]+(1-q) \bar{a}(s)\left(\frac{s^{\alpha}}{(1-q)^{\alpha}} L_{q}\left[u_{n}\right]\right. \\
& \left.-\sum_{i=0}^{m-1} \frac{d_{q}^{i} u_{n}(0)}{d_{q} t^{i}} \frac{s^{\alpha-i-1}}{(1-q)^{\alpha-i}}+L_{q}\left[f\left(t, u_{n}\right)\right]\right),
\end{aligned}
$$

where $\bar{a}(s)$ is the Laplace transform of some function.

Considering $L_{q}\left[f\left(t, u_{n}\right)\right]$ as a restricted variation so that after taking the classical variational derivative to both sides of (56), we can obtain

$$
\delta L_{q}\left[u_{n+1}\right]=\delta L_{q}\left[u_{n}\right]+\bar{a}(s) \frac{s^{\alpha}}{(1-q)^{\alpha-1}} \delta L_{q}\left[u_{n}\right],
$$

from which we can derive

$$
\bar{a}(s)=-\frac{(1-q)^{\alpha-1}}{s^{\alpha}} .
$$

The inverse Laplace transform of $\bar{a}(s)$ is

$$
a(t)=-\frac{t^{\alpha-1}}{\Gamma_{q}(\alpha)} .
$$

As a result, we can get

$$
\lambda(t, \tau)=-1 .
$$


Substituting $\lambda(t, \tau)=-1$ into (54), the variational iteration formula is determined as

$$
u_{n+1}=u_{n}-{ }_{q} I_{0}^{\alpha}\left[{ }_{q}^{C} D_{0}^{\alpha} u_{n}+f\left(\tau, u_{n}\right)\right], \quad 0<\alpha .
$$

Example 5.8 Now consider the application in the initial value problems of the Caputo $q$-fractional difference equations [12],

$$
{ }_{q}^{C} D_{a}^{\alpha} u=\omega u+f(t), \quad u(a)=c, \quad t \in T_{q}^{\alpha}, 0<\alpha<1 .
$$

We have the following variational iteration formula:

$$
u_{n+1}=u_{n}-\int_{a}^{t} \frac{(t-q \tau)^{(\alpha-1)}}{\Gamma_{q}(\alpha)}\left({ }_{q}^{C} D_{a}^{\alpha} u_{n}-\omega u_{n}-f(\tau)\right) d_{q} \tau .
$$

Starting from the initial iteration

$$
u_{0}=u(a)=c
$$

the successive solutions can be given as

$$
\begin{aligned}
u_{1}= & c\left[1+\frac{\omega(t-a)^{(\alpha)}}{\Gamma_{q}(\alpha+1)}\right]+{ }_{q} I_{a}^{\alpha} f(t), \\
u_{2}= & c\left[1+\frac{\omega(t-a)^{(\alpha)}}{\Gamma_{q}(\alpha+1)}+\frac{\omega^{2}(t-a)^{(2 \alpha)}}{\Gamma_{q}(2 \alpha+1)}\right]+{ }_{q} I_{a}^{\alpha} f(t)+\omega_{q} I_{a}^{2 \alpha} f(t), \\
& \vdots \\
u_{n}= & c \sum_{k=0}^{n} \frac{\omega^{k}(t-a)^{(k \alpha)}}{\Gamma_{q}(k \alpha+1)}+\int_{a}^{t}(t-q \tau)^{(\alpha-1)}\left[\sum_{k=0}^{n} \frac{\omega^{k}\left(t-q^{\alpha} \tau\right)^{(k \alpha)}}{\Gamma_{q}(k \alpha+\alpha)}\right] f(\tau) d_{q} \tau .
\end{aligned}
$$

For $n \rightarrow \infty, u_{n}$ tends to the exact solution

$$
u=c_{q} E_{\alpha}(\omega,(t-a))+\int_{a}^{t}(t-q \tau)^{(\alpha-1)}{ }_{q} E_{\alpha, \alpha}\left(\omega, t-q^{\alpha} \tau\right) f(\tau) d_{q} \tau
$$

where ${ }_{q} E_{\alpha, \beta}\left(\omega,\left(t-q^{\alpha} \tau\right)\right)$ is the discrete Mittag-Leffler function defined by [12]

$$
{ }_{q} E_{\alpha, \beta}\left(\omega, t-q^{\alpha} \tau\right)=\sum_{k=0}^{\infty} \frac{\omega^{k}\left(t-q^{\alpha} \tau\right)^{(k \alpha)}}{\Gamma_{q}(k \alpha+\beta)}
$$

and

$$
{ }_{q} E_{\alpha, 1}(\omega,(t-a))={ }_{q} E_{\alpha}(\omega, t-a) .
$$

Readers are referred to the recent development in the application of the VIM for solving fuzzy equations [41-43] and the calculus of variations on time scales [44-48]. Since this study only concentrates on the applications of the VIM, other numerical methods in FC can be found in [49-51]. 


\section{Conclusions}

We aim at some new applications of the VIM from differential equations to $q$-fractional difference equations, and the following main contributions of this study are obtained:

(a) Designing a maple program of the VIM for differential equations. Now, there is no need for one to obtain approximate solutions of high order by hand. The efficiency and accuracy are improved;

(b) Correcting the popularly used variational iteration formulae in FC and explicitly identifying some new Lagrange multipliers from the Laplace transform. The FDEs are transformed into generalized Volterra integral equations;

(c) Applying the VIM in $q$-difference equations and identifying a Lagrange multiplier of $q$-difference equations of $m$ th order;

(d) Extending the VIM to FQC and investigating the initial value problems analytically. The obtained variational iteration formula in FQC can reduce to those in FC and QC.

Due to the rapid development of advanced applied sciences, non-classical tools of calculus, i.e., fractional calculus, $q$-calculus, etc., have been becoming more active and have been found useful in describing important physical phenomena. This study discusses some new applications of the VIM and provides a potential tool to analytically investigate such models. There is still some other work needed to consider, i.e., maple-packages or the symbolic computation of the VIM in FC even in FQC, other numerical methods based on the VIM, etc. The authors believe, in not far future, the VIM can play the same crucial role as that in ordinary calculus.

\section{Competing interests}

The authors declare that they have no competing interests.

Authors' contributions

All authors read and approved the final manuscript.

\section{Author details}

${ }^{1}$ College of Mathematics \& Information Science, Neijiang Normal University, Neijiang, 641112, China. ${ }^{2}$ College of Water Resources and Hydropower, Sichuan University, Chengdu, 610065, China. ${ }^{3}$ Department of Mathematics and Computer Sciences, Cankaya University, Balgat, Ankara 06530, Turkey. ${ }^{4}$ Institute of Space Sciences, Magurele, Bucharest, Romania.

${ }^{5}$ Department of Mathematics, King Abdulaziz University, Jeddah, Saudi Arabia.

\section{Acknowledgements}

The authors would like to express their deep gratitude to the referees for their valuable suggestions and comments. The work is financially supported by the NSFC (11061028) and the key program of the NSFC (51134018).

Received: 11 September 2012 Accepted: 5 January 2013 Published: 24 January 2013

\section{References}

1. Saxena, R, Yadav, R, Purohit, S: Kober fractional $q$-integral operator of the basic analogue of the H-function. Rev. Téc. Fac. Ing., Univ. Zulia 28, 154-158 (2005)

2. Rajković, PM, Marinković, SD, Stanković, MS: Fractional integrals and derivatives in q-calculus. Appl. Anal. Discrete Math. 1, 311-323 (2007)

3. Mansour, Z: Linear sequential q-difference equations of fractional order. Fract. Calc. Appl. Anal. 12, 159-178 (2009)

4. Atici, FM, Senguel, S: Modeling with fractional difference equations. J. Math. Anal. Appl. 369, 1-9 (2010)

5. Herrmann, R: Common aspects of $q$-deformed Lie algebras and fractional calculus. Physica A 389, 4613-4622 (2010)

6. Purohit, SD, Yadav, RK: On generalized fractional $q$-integral operators involving the $q$-Gauss hypergeometric function. Bull. Math. Anal. Appl. 2, 35-44 (2010)

7. Atici, FM, Eloe, PW: Linear systems of fractional nabla difference equations. Rocky Mt. J. Math. 41, 353-370 (2011)

8. Al-Salam, WA: Some fractional q-integrals and q-derivatives. Proc. Edinb. Math. Soc. 15, 135-140 (1966)

9. Agarwal, R: Certain fractional q-integrals and q-derivatives. Proc. Camb. Philos. Soc. 66, 365-370 (1969)

10. Al-Salam, W, Verma, A: A fractional Leibniz q-formula. Pac. J. Math. 60, 1-9 (1975)

11. Mozyrska, D, Pawłuszewicz, E: Observability of linear q-difference fractional-order systems with finite initial memory. Bull. Pol. Acad. Sci. 58, 601-605 (2010)

12. Abdeljawad, T, Baleanu, D: Caputo $q$-fractional initial value problems and a $q$-analogue Mittag-Leffler function. Commun. Nonlinear Sci. Numer. Simul. 16, 4682-4688 (2011) 
13. Ferreira, RAC: Positive solutions for a class of boundary value problems with fractional $q$-differences. Comput. Math. Appl. 61, 367-373 (2011)

14. Ahmad, B, Nieto, Jj: On nonlocal boundary value problems of nonlinear q-difference equations. Adv. Differ. Equ. 2012, 81 (2012)

15. Ahmad, B, Ntouyas, SK, Purnaras, IK: Existence results for nonlocal boundary value problems of nonlinear fractional q-difference equations. Adv. Differ. Equ. 2012, 140 (2012)

16. Graef, JR, Kong, L: Positive solutions for a class of higher order boundary value problems with fractional q-derivatives. Appl. Math. Comput. 218, 9682-9689 (2012)

17. $\mathrm{He}, \mathrm{JH}$ : Approximate analytical solution for seepage flow with fractional derivatives in porous media. Comput. Methods Appl. Mech. Eng. 167, 57-68 (1998)

18. He, JH: Variational iteration method - a kind of non-linear analytical technique: some examples. Int. J. Non-Linear Mech. 34, 699-708 (1999)

19. He, JH: Variational iteration method - some recent results and new interpretations. J. Comput. Appl. Math. 207, 3-17 (2007)

20. $\mathrm{He}, \mathrm{JH}, \mathrm{Wu}, \mathrm{XH}$ : Variational iteration method: new development and applications. Comput. Math. Appl. 54, 881-894 (2007)

21. El-Tawil, MA, Bahnasawi, AA, Abdel-Naby, A: Solving Riccati differential equation using Adomian's decomposition method. Appl. Math. Comput. 157, 503-514 (2004)

22. Hull, T, Enright, W, Fellen, B, Sedgwick, A: Comparing numerical methods for ordinary differential equations. SIAM J. Numer. Anal. 9, 603-637 (1972)

23. Kac, VG, Cheung, P: Quantum Calculus. Springer, Berlin (2002)

24. Bohner, M, Peterson, AC: Advances in Dynamic Equations on Time Scales. Birkhäuser, Basel (2003)

25. Gasper, G, Rahman, M: Basic Hypergeometric Series. Cambridge University Press, Cambridge (2004)

26. Jackson, FH: q-form of Taylor's theorem. Messenger Math. 38, 62-64 (1909)

27. Jackson, FH: On q-definite integrals. Q. J. Pure Appl. Math. 41, 193-203 (1910)

28. Wu, GC: Variational iteration method for $q$-diffusion equations on time scales. Heat Transf. Res. (2012, in press)

29. Wu, GC: Variational iteration method for q-difference equations of second order. J. Appl. Math. 2012, 102850 (2012)

30. Kong, H, Huang, LL: Lagrange multipliers of $q$-difference equations of third order. Commun. Fract. Calc. 3, 30-33 (2012)

31. Podlubny, I: Fractional Differential Equations. Academic Press, San Diego (1999)

32. Kilbas, AA, Srivastav, HM, Trujillo, JJ: Theory and Applications of Fractional Differential Equations. Elsevier, Amsterdam (2006)

33. Sheng, $\mathrm{H}, \mathrm{Li}, \mathrm{Y}, \mathrm{Chen}, \mathrm{YQ}$ : Application of numerical inverse Laplace transform algorithms in fractional calculus. J. Franklin Inst. 348, 315-330 (2011)

34. Wu, GC: Variational iteration method for the fractional diffusion equations in porous media. Chin. Phys. B 21, 120504 (2012)

35. Wu, GC, Baleanu, D: Variational iteration method for the Burgers' flow with fractional derivatives - new Lagrange multipliers. Appl. Math. Model. (2012, in press). doi:10.1016/j.apm.2012.12.018

36. Baleanu, D, Golmankhaneh, AK, Golmankhaneh, AK: Solving of the nonlinear and linear Schrödinger equations by the homotopy perturbation method. Rom. J. Phys. 54, 823-832 (2010)

37. Diethelm, K: The Analysis of Fractional Differential Equations. Springer, Berlin (2010)

38. Atici, FM, Eloe, PW: Fractional q-calculus on a time scale. J. Nonlinear Math. Phys. 14, $341-352$ (2007)

39. Hahn, W: Beiträge zur Theorie der Heineschen Reihen. Die 24 Integrale der hypergeometrischen q-Differenzengleichung. Das q-Analogon der Laplace-Transformation. Math. Nachr. 2, 340-379 (1949)

40. Annaby, MH, Mansour, ZSI: q-Fractional Calculus and Equations. Springer, Berlin (2012)

41. Allahviranloo, $\mathrm{T}, \mathrm{Abb}$ asbandy, $\mathrm{S}$, Rouhparvar, $\mathrm{H}$ : The exact solutions of fuzzy wave-like equations with variable coefficients by a variational iteration method. Appl. Soft Comput. 11, 2186-2192 (2011)

42. Jafari, H, Saeidy, M, Baleanu, D: The variational iteration method for solving $n$-th order fuzzy differential equation. Cent. Eur. J. Phys. 10, 76-85 (2012)

43. Jafari, $\mathrm{H}$, Khalique, $\mathrm{CM}$ : Homotopy perturbation and variational iteration methods for solving fuzzy differential equations. Commun. Fract. Calc. 3, 38-48 (2012)

44. Bangerezako, G: Variational q-calculus. J. Math. Anal. Appl. 289, 650-665 (2004)

45. Ferreira, RAC, Torres, DFM: Higher-order calculus of variations on time scales. Math. Control Theory Finance 2008 $149-159$ (2008)

46. Abdeljawad, T, Jarad, F, Baleanu, D: Variational optimal-control problems with delayed arguments on time scales. Adv. Differ. Equ. 2009, 840386 (2009)

47. Martins, N, Torres, DFM: Calculus of variations on time scales with nabla derivatives. Nonlinear Anal., Theory Methods Appl. 71, e763-e773 (2009)

48. Malinowska, AB, Torres, DFM: The Hahn quantum variational calculus. J. Optim. Theory Appl. 147, 419-442 (2010)

49. Chen, W, Sun, HG, Li, XC: Mechanics Engineering Problems of Fractional Derivative Modeling. Science Press, Beijing (2010)

50. Baleanu, D, Diethelm, K, Scalas, E, Trujillo, Jj: Fractional Calculus Models and Numerical Methods. World Scientific Singapore (2012)

51. Duan, JS, Buleanu, D, Wazwaz, AM: A review of the Adomian decomposition method and its applications to fractional differential equations. Commun. Fract. Calc. 3, 73-99 (2012)

doi:10.1186/1687-1847-2013-21

Cite this article as: Wu and Baleanu: New applications of the variational iteration method - from differential equations to $q$-fractional difference equations. Advances in Difference Equations 2013 2013:21. 